\title{
A Comparative Analysis of the Gini Index
}

\section{Romina Mahinpei \\ University of British Columbia}

ABSTRACT: Around the world, the Gini index is used to represent income inequality and is compared between regions. Proposed by Corrado Gini in 1912, the index summarizes the income disparity of an area into a single value that falls between zero and one [1]. There are numerous methods for evaluating the Gini index [2]. Considering its global use, it is essential for these different approaches to provide consistent results for a region. This paper compares the Gini indices obtained using three of the earliest developed methods. These methods include Gini's original method, the relative mean difference method, and the geometric method. The geometric method, specifically, can be applied either algebraically or geometrically. In this report these three approaches were applied to the 2017 Canadian income distribution from Statistics Canada. To ensure a fair analysis, the methods were also applied to the Canadian income distributions from 1999 and 2010, with their calculations being summarized in Appendices A and B respectively.From the investigation, it was discovered that Gini's original method and the relative mean difference method, (collectively referred to as the algebraic methods), provided identical results for all three data sets. However, the geometric methods, referring to the Trapezoid Rule and Logger Pro technology, provided values that differed from one another and the algebraic methods. This highlights the importance of acknowledging the method used to derive the Gini Index to ensure consistency and to allow a valid interpretation. The results of this paper also suggest that the algebraic methods should be preferred over the geometric methods when dealing with discrete data to ensure consistent results.

KEYWORDS: Gini index, income distribution, inequality

\section{INTRODUCTION}

The Gini index, proposed by Corrado Gini in 1912, is a common measure of income distribution [1]. Globally, the Gini index is also used to measure other distributions, including that of consumption and wealth, but it is most widely recognized for measuring income distribution [2]. Even though there are multiple ways to represent the measure of a region's income inequality, the Gini index is preferred since it condenses income inequality into a single value that falls between zero and one [2]. A low Gini value indicates a low level of income disparity, while a high Gini value indicates a high level of income disparity [1]. The Gini indices of countries are determined and compared globally, with South Africa having one of the largest Gini indices at 0.630 and Slovenia having one of the smallest indices at 0.242 [3]. Due to its global use, the Gini index has become one of the most popular statistical indices and is used to quantify the social and economic conditions of a nation [4].

There are multiple methods for the evaluation of the Gini
Index. Recent studies have claimed there to be a minimum of 13 formulations for the index [1]. Ever since its first proposal in Corrado Gini's book, Variabilita e Mutabilita (1912), various methods have been developed for the evaluation of the Gini index [1]. Three of the earliest methods include Gini's original approach, the relative mean difference approach, and the geometric approach [2]. It is important for these methods to provide the same value to prevent confirmation bias that may result from the selection of method that provides the most desirable result. Thus, to test whether the three methods provide the same results, this paper answers the following research question: How do the Gini values obtained by Gini's original method, the relative mean difference method, and the geometric method compare to one another? The three approaches are applied to the 2017 Canadian income distribution from Statistics Canada. Appendices A and B also include the calculations for the Canadian income distribution in 1999 and 2010 using the raw data from Statistics Canada to allow for a better analysis of the methods. 


\section{METHODS}

\section{Development of Methods}

In this section, discrete data consisting of individual data points will be used to compare the three methods rather than continuous data. This is because the Gini index is used with real-life situations, which often involve individual data points. In total, there are $n$ data points, excluding the origin $(0,0)$, that represent the proportion of income held by a proportion of the population. Note that the $n$ data points also provide the total number of individuals in the population. The x-coordinate of each point represents the cumulative proportion of the population up to the $k t h$ individual as shown:

$$
x_{p}=\frac{k}{n}
$$

where:

$$
\begin{aligned}
& x_{p}=\text { cumulative proportion of population up to the } \\
& k t h \text { individual } \\
& k=\text { number of individuals up to the } k t h \text { individual } \\
& n=\text { total number of individuals (i.e. data points) }
\end{aligned}
$$

The $y$-coordinate of each point represents the proportion of income held by the $k t h$ individual and anyone before them. Consider the given sequence defining the income of the $k t h$ individual in a population of $\boldsymbol{n}$ individuals. Note that the sequence is arranged in a non-strictly increasing order such that $Y_{1} \leq Y_{2} \leq \ldots \leq Y_{n}$.

$$
\left\{Y_{1}, Y_{2}, Y_{3}, \ldots, Y_{n}\right\}
$$

The cumulative proportion of the income up to the $k t h$ individual is then:

$$
y_{p}=\frac{\sum_{i=1}^{k} Y_{i}}{I}
$$

where:

$$
\begin{aligned}
& y_{p}=\text { cumulative proportion of income up to the } k t h \\
& \text { individual } \\
& I=\text { total income of the region }
\end{aligned}
$$

When evaluating the Gini index, the cumulative proportion of population (the $\mathrm{x}$-coordinate) is obtained using equation 1.1, and the cumulative proportion of income (the y-coordinate) is obtained using equation 1.2. Note that both the $x$ and $y$-coordinates of the data points are decimal values between 0.0 and 1.0 inclusive. For further clarification, refer to Appendix C to see the step-by-step evaluation of the $\mathrm{x}$ and $\mathrm{y}$-coordinates for an arbitrary population.

Using the established parameters, the following sections introduce three of the earliest methods for evaluating the Gini Index. Note that examples of applying the defined methods are later provided in Section III and in Appendices A \& B.

\section{Gini's Original Approach}

Gini's index was introduced to the anglophone world by Hugh Dalton in 1920 and was originally expressed using Gini's mean difference [5]. As explained by Dalton, Gini's mean difference $(\Delta)$ is the "arithmetic average of the differences, taken positively, between all possible pairs of incomes" [6]. Mathematically, this is expressed using equation 2.1.

$$
\Delta=\frac{2}{n^{2}} \sum_{i=1}^{\frac{n+1}{2}}(n+1-2 i)\left(y_{n-i+1}-y_{i}\right)
$$

where:

$$
\begin{gathered}
n=\text { an odd whole number representing the total } \\
\text { number of individuals } \\
y_{i}=\text { the cumulative proportion of income up to } i t h \\
\text { individual }
\end{gathered}
$$

Note that Appendix $D$ includes the steps that prove the equality between Dalton's statement and the mathematical definition of $\Delta$. From translated extracts of the Italian text, the Gini index is defined as Gini's mean difference divided by twice the arithmetic mean $\left(\mu_{y}\right)[4]$.

$$
G=\frac{\Delta}{2 \mu_{y}}
$$

After substituting equation 2.1 for $\Delta$ in 2.2 , the original definition of the index is obtained in equation 2.3 .

$$
G=\frac{\Delta}{2 \mu_{y}}=\frac{1}{\mu_{y} n^{2}} \sum_{i=1}^{\frac{n+1}{2}}(n+1-2 i)\left(y_{n-i+1}-y_{i}\right)
$$

A major limitation of the original definition is that it considers $n$ to be an odd number. To overcome this restriction, Gini proposed an adjusted version of the equation in 1914 that allows $\boldsymbol{n}$ to be any whole number [4]. Within translated extracts of the document, the adjusted definition is expressed using equation 2.4. 


$$
G=\frac{1}{2 \mu_{y} n^{2}} \sum_{i=1}^{n}(n+1-2 i)\left(y_{n-i+1}-y_{i}\right)
$$

where:

$$
\begin{aligned}
& n=\text { total number of individuals } \\
& y_{i}=\text { the cumulative proportion of income up to ith } \\
& \quad \text { individual } \\
& \mu_{y}=\text { the arithmetic mean of the cumulative } \\
& \text { proportions of income }
\end{aligned}
$$

As proven in Appendix E, the original and adjusted definitions are interchangeable with one another, with both forms only being applicable to discrete data. Their only difference is that the adjusted version is inclusive of all whole numbers, making it preferable over the original version.

\section{Relative Mean Difference Approach}

Following the original approach, Maurice Kendall and Alan Stuart defined the Gini index using the relative mean difference $(R M D)$ considering its popularity as a statistical measure at the time [2]. Kendall and Stuart defined the Gini index as "one-half of the coefficient of concentration" [7], with the coefficient of concentration being known today as the relative mean difference. Mathematically, the relative mean difference is the absolute mean difference $(A M D)$ over the arithmetic mean [2].

$$
R M D=\frac{A M D}{\mu}
$$

$A M D$ is the arithmetic mean of the absolute value of all possible differences between the data points in a set [2]. Let $y$ represent the cumulative proportions of income for a population. The sum of the absolute value of all the possible differences between the possible pairs of $y$ values is then expressed using sigma notation.

$$
\sum_{i=1}^{n} \sum_{j=1}^{n}\left|y_{i}-y_{j}\right|
$$

To find the arithmetic mean of the absolute value of all possible differences, expression 3.2 is divided by the number of terms in the summation. Since there are $n$ possible cumulative proportions of income and the differences between the $n$ possible values are taken, the summation has a total of $n^{2}$ terms. The absolute mean difference is then written as an equation.

$$
A M D=\frac{1}{n^{2}} \sum_{i=1}^{n} \sum_{j=1}^{n}\left|y_{i}-y_{j}\right|
$$

By substituting equation 3.3 for $A M D$ in equation 3.1, a formula for the relative mean difference is obtained.

$$
R M D=\frac{A M D}{\mu}=\frac{\sum_{i=1}^{n} \sum_{j=1}^{n}\left|y_{i}-y_{j}\right|}{n^{2} \mu_{y}}
$$

As explained by Kendall and Stuart, the Gini index is equal to one-half of the relative mean difference [7].

$$
G=\frac{R M D}{2}
$$

Substituting equation 3.4 for $R M D$ in equation 3.5 then provides the Gini index.

$$
G=\frac{R M D}{2}=\frac{\sum_{i=1}^{n} \sum_{j=1}^{n}\left|y_{i}-y_{j}\right|}{2 n^{2} \mu_{y}}
$$

where:

$$
\begin{aligned}
& \begin{array}{l}
n=\text { total number of individuals } \\
y_{i}=\text { the cumulative proportion of income up to } i t h \\
\text { individual }
\end{array} \\
& y_{j}=\text { the cumulative proportion of income up to } j \text { th } \\
& \text { individual } \\
& \mu_{y}=\text { the arithmetic mean of the cumulative } \\
& \text { proportions of income }
\end{aligned}
$$

Similar to the original method, the relative mean difference approach is only applicable to discrete data as it requires the substitution of the data points' coordinates.

\section{Geometric Approach}

The geometric approach to evaluating the Gini index was developed by Alan Hickrod and made public by Raymond Lows. In his publication, Lows describes the Gini coefficient as "a unique numerical value which is used as an index of inequality associated with a particular Lorenz Curve" [8]. The Lorenz Curve is a graphical representation of wealth distribution developed by Max Lorenz that plots the cumulative proportion of population on the $x$-axis and the cumulative 
proportion of income on the $y$-axis. By connecting the points, a curve representing the wealth distribution is created as shown in Figure 1. A population where wealth is distributed equally creates a straight line, referred to as the line of equality $\boldsymbol{y}=\boldsymbol{x}$, and unequal distribution results in a curve, referred to as the Lorenz curve [9].

For the arbitrary data set in Table 1, the following Lorenz curve is created.

It is important to denote that the endpoints of a Lorenz curve, regardless of the extent of inequality, are always $(0,0)$ and $(1,1)$ since zero individuals will have zero income, and all of the individuals will have all of the income. Thus, the domain and range of a Lorenz curve can be determined.

$$
\text { Domain }=\{x \mid 0 \leq x \leq 1\}
$$$$
\text { Range }=\{y \mid 0 \leq y \leq 1\}
$$

Lows discusses the possibility of calculating the Gini index using "the area below the line of absolute equality bounded by the curve of unequal distribution", referred to as area of $A$, and the total area below the line of absolute equality [8], referred to as the area of $A$ plus $B$.

Using the areas illustrated above, Lows defined the Gini index as the ratio of area $A$ to the area of $A$ and $B$ to obtain the following equation.

$$
G=\frac{A}{A+B} \quad \text { [8] }
$$

where:

$A=$ the area between the Lorenz curve and the line of equality

$B=$ the area under the Lorenz curve

Since $A+B$ is equal to the area of a triangle with a length of 1 unit and a height of 1 unit, the following must be true.

$$
A+B=\frac{1}{2} \times 1 \times 1=\frac{1}{2}
$$

Substituting equation 4.2 into equation 4.1 then gives the Gini index in terms of $A$.

$$
G=\frac{A}{A+B}=\frac{A}{\frac{1}{2}}=2 A
$$

Since $B$ is the area under the Lorenz Curve and can be easily evaluated using a definite integral, the Gini index is
Table 1. An arbitrary data set for the cumulative proportion of population and the corresponding cumulative proportion of income.

\section{Cumulative Proportion of Population}

(x)

0.00

0.20

0.40

0.60

0.80

1.00

\section{Cumulative Proportion of} Income

(y)

0.00

0.04

0.16

0.36

0.64

1.00

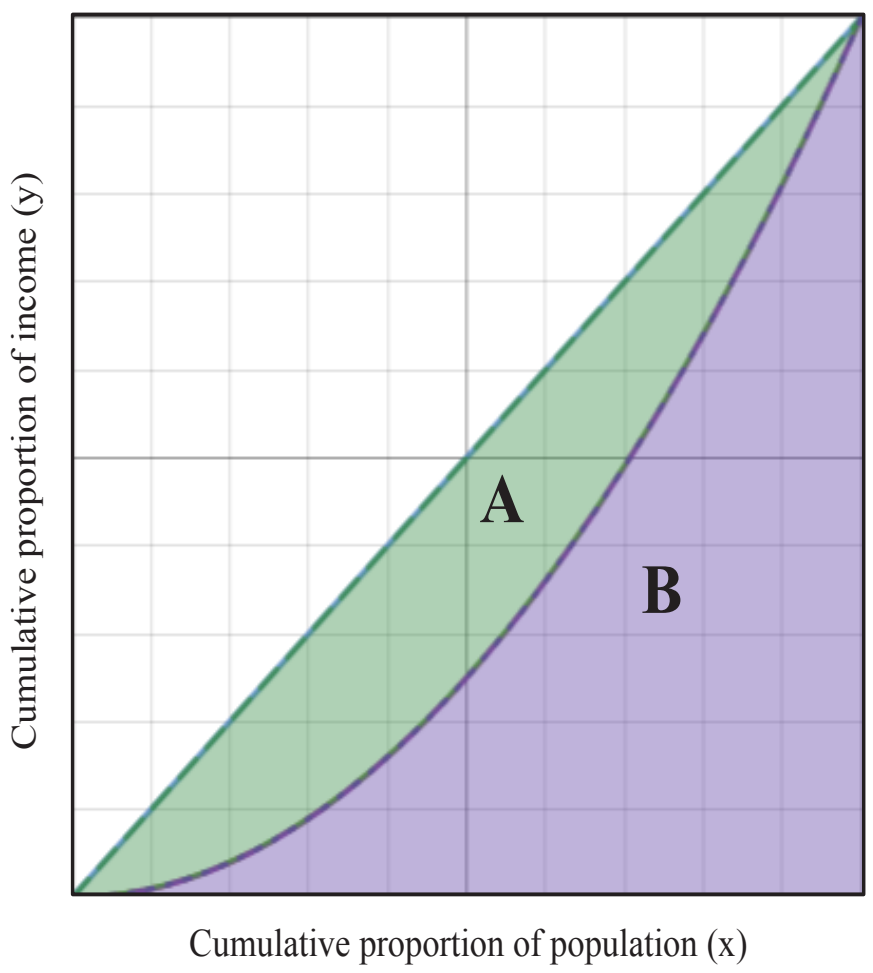

Figure 1. The line of equality and the Lorenz Curve for the arbitrary data in Table 1.

more commonly written in terms of $B$. Using equation 4.2, $A$ is written in terms of $B$ and is then used to rewrite the Gini index in terms of $B$. 


$$
\begin{gathered}
A+B=\frac{1}{2} \\
A=\frac{1}{2}-B \\
G=2 A=2\left(\frac{1}{2}-B\right)=1-2 B
\end{gathered}
$$

Since $B$ is the area under the Lorenz curve $L(x)$, equation 4.4 can be written using a definite integral, as is commonly done [2].

$$
G=1-2 \int_{0}^{1} L(x) d x
$$

where:

$$
L(x)=\text { Lorenz curve of the region under study }
$$

One of the major benefits of the geometric method is its applicability to both discrete and continuous data. Out of the three discussed methods, only the geometric approach can be used with continuous data. However, this method does require an approximation for the Lorenz Curve when dealing with discrete data, which can decrease the accuracy of the evaluated Gini index.

\section{Application of Methods}

To determine whether the three methods provide the same values, each approach is applied to the 2017 Canadian income distribution provided in Table 2 . Each quintile in the data represents one of the five equal groups the total population has been divided into based on income. The first quantile has the lowest-income and an average income of $\$ 26,513$ and the fifth quantile having the highest-income and an average income of $\$ 164,117$ [10]. The cumulative proportions of population have already been evaluated by Statistics Canada using equation 1.1. As an example, the first quintile has its cumulative proportion of population equal to 0.2 , the second quintile has $\mathbf{0 . 4}$, and so on until the last quintile, with a cumulative proportion of population that is equal to 1.0 .
Table 2. 2017 Canadian income distribution [10]

$\begin{array}{cccc}\begin{array}{c}\text { Income } \\ \text { Quintile }\end{array} & \begin{array}{c}\text { Cumulative } \\ \text { Proportion of } \\ \text { Population } \\ \boldsymbol{x}_{\boldsymbol{p}}\end{array} & \begin{array}{c}\text { Proportion of } \\ \text { Total Income }\end{array} & \begin{array}{c}\text { Cumulative } \\ \text { Proportion of } \\ \text { Income }\end{array} \\ 1^{\text {st }} \text { Quintile } & 0.2 & 0.067 & \boldsymbol{y}_{\boldsymbol{p}} \\ 2^{\text {nd }} \text { Quintile } & 0.4 & 0.124 & 0.067 \\ 3^{\text {rd }} \text { Quintile } & 0.6 & 0.166 & 0.357 \\ 4^{\text {th }} \text { Quintile } & 0.8 & 0.229 & 0.586 \\ 5^{\text {th }} \text { Quintile } & 1.0 & 0.414 & 1.000 \\ & & & \end{array}$

Using the second and third columns of Table 2, the cumulative proportion of income is obtained, with the results summarized in the fourth column of Table 2.

$$
\begin{gathered}
\text { 1st Quintile: } y_{1}=\frac{\sum_{i=1}^{1} y_{i}}{Y}=\frac{0.067}{1.000}=0.067 \\
\text { 2nd Quintile: } y_{2}=\frac{\sum_{i=1}^{2} y_{i}}{Y}=\frac{0.067+0.124}{1.000}=0.191 \\
\text { 3rd Quintile: } y_{3}=\frac{\sum_{i=1}^{3} y_{i}}{Y}=\frac{0.067+0.124+0.166}{1.000}=0.357 \\
\text { 4th Quintile: } y_{4}=\frac{\sum_{i=1}^{4} y_{i}}{Y}=\frac{0.067+0.124+0.166+0.229}{1.000}=0.586 \\
\text { 5th Quintile: } y_{5}=\frac{\sum_{i=1}^{5} y_{i}}{Y}=\frac{0.067+0.124+0.166+0.229+0.414}{1.000}=1.000
\end{gathered}
$$

For the application of each method, the adjusted data from the second and fourth columns of Table 2 are used. Note that Appendices A and B include the data, calculations, and Gini indices for two additional data sets from 1999 and 2010.

\section{Gini's Original Approach}

To apply Gini's original approach, equation 2.4 is used. As shown in Appendix $F$, the original equation, which provides the same Gini index as below, can also be used in this scenario since there are an odd number of data points.

$$
G=\frac{1}{2 \mu_{y} n^{2}} \sum_{i=1}^{n}(n+1-2 i)\left(y_{n-i+1}-y_{i}\right)
$$


Using the data from Table 2:

$$
\begin{gathered}
n=5 \\
\mu_{y}=\frac{0.067+0.191+0.357+0.586+1.000}{5}=0.4402
\end{gathered}
$$

The values above are then substituted into equation 2.4, and the Gini index is obtained.

$$
\begin{gathered}
G=\frac{1}{2 \mu_{y} n^{2}} \sum_{i=1}^{n}(n+1-2 i)\left(y_{n-i+1}-y_{i}\right) \\
G=\frac{1}{22.01} \sum_{i=1}^{6}(6-2 i)\left(y_{6-i}-y_{i}\right) \\
G=\frac{1}{22.01}[4(1.000-0.067)+2(0.586-0.191) \\
+0-2(0.191-0.586)-4(0.067-1.000)] \\
G=\frac{9.044}{22.01}[3.732+0.790+0+0.790+3.732] \\
\therefore \boldsymbol{G} \approx \mathbf{0 . 4 1 1}
\end{gathered}
$$

\section{Relative Mean Difference Approach}

For the relative mean difference approach, equation 3.6 is used.

$$
G=\frac{\sum_{i=1}^{n} \sum_{j=1}^{n}\left|x_{i}-y_{j}\right|}{2 n^{2} \mu_{y}}
$$

The absolute mean difference (i.e., the numerator in equation 3.6) is at first evaluated. The corresponding calculations for this process are summarized in Appendix G.

$$
\begin{gathered}
A M D=\sum_{i=1}^{n} \sum_{j=1}^{n}\left|x_{i}-y_{j}\right| \\
\therefore A M D=9.044
\end{gathered}
$$

The value of the absolute mean difference is then substituted into equation 3.6 along with $\mu_{y}=0.4402$ and the Gini index is obtained

$$
\begin{gathered}
G=\frac{\sum_{i=1}^{n} \sum_{j=1}^{n}\left|x_{i}-y_{j}\right|}{2 n^{2} \mu_{y}} \\
G=\frac{9.044}{2 \times 25 \times 0.4402}
\end{gathered}
$$

$$
\therefore G \approx 0.411
$$

\section{Geometric Approach}

To apply the geometric approach, equation 4.6 is used.

$$
G=1-2 \int_{0}^{1} L(x) d x
$$

Considering that the data used is discrete, a Lorenz curve $L(x)$ approximates for the data, leading to an approximation for the area under the curve. In this paper, two common methods of approximation, Trapezoid Rule and Logger Pro technology, are used.

The Trapezoid Rule is an algebraic method of approximating a definite integral [11]. It divides the curve into $n$ partitions so each contains a trapezoid. The definite integral is then approximated by summing the areas of all the trapezoids under the curve [11]. In comparison to the more common Riemann's approximation method, the Trapezoid Rule provides more accurate results. Riemann's method uses rectangles, which are less fit to the area under a curve when compared to trapezoids as show in Figure 3. Considering this advantage, the Trapezoid Rule is the chosen method for algebraically approximating a definite integral.

Nonetheless, it is important to acknowledge that the approximation of a definite integral can be derived using approaches other than the Trapezoid Rule and Logger Pro technology. Various other methods involving approximations, such as the Midpoint Rule or Simpson's Rule, can be used [11]. The two approaches were selected based on their higher degrees of accuracy and their accompanying graphical representations that allow for a better understanding.

Trapezoid Rule. Using Table 2, corresponding data points are plotted, and trapezoids are drawn to approximate the area under $L(x)$ as shown in Figure 4.

The definite integral $\int_{0}^{1} L(x) d x$ is approximated by summing the areas of the trapezoids. The area of a trapezoid, 

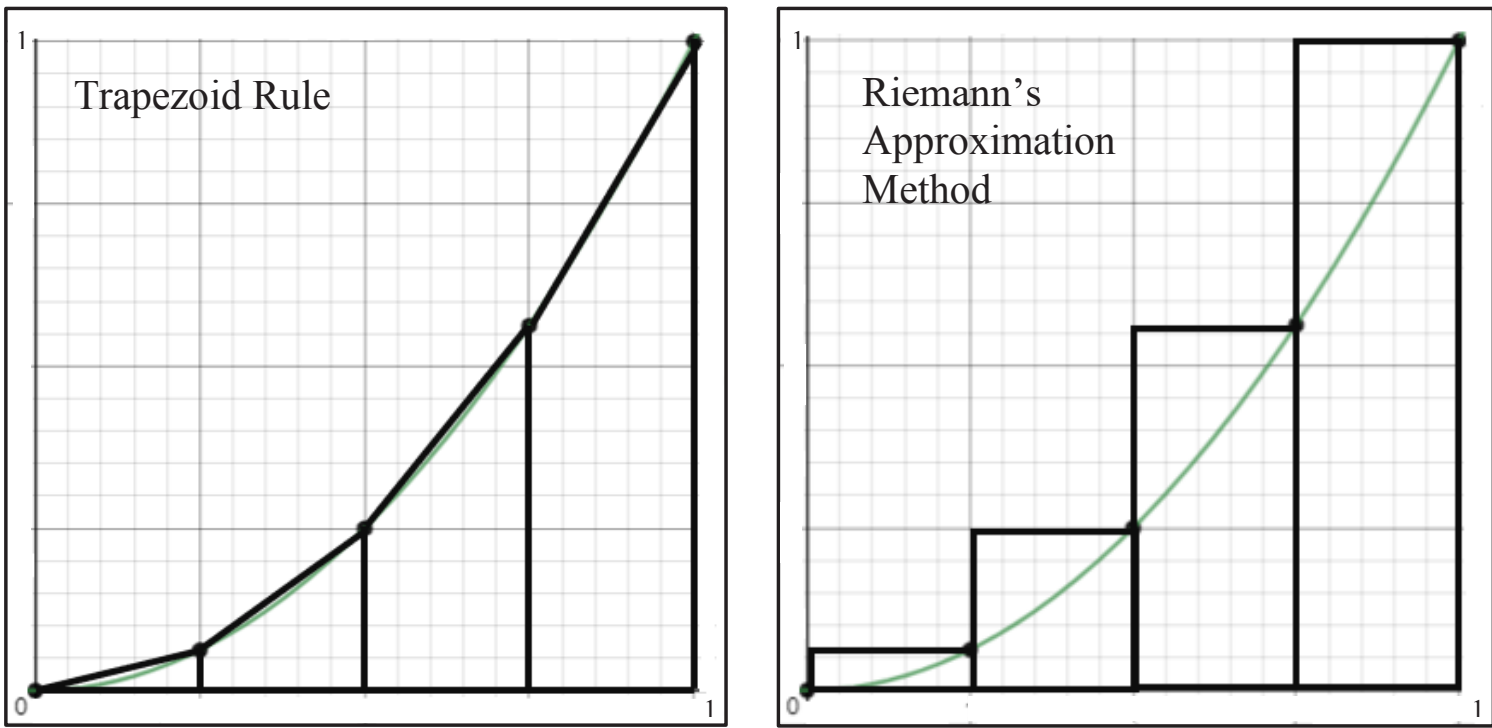

Figure 3. Graphical representation of Trapezoid Rule and Riemann's method.
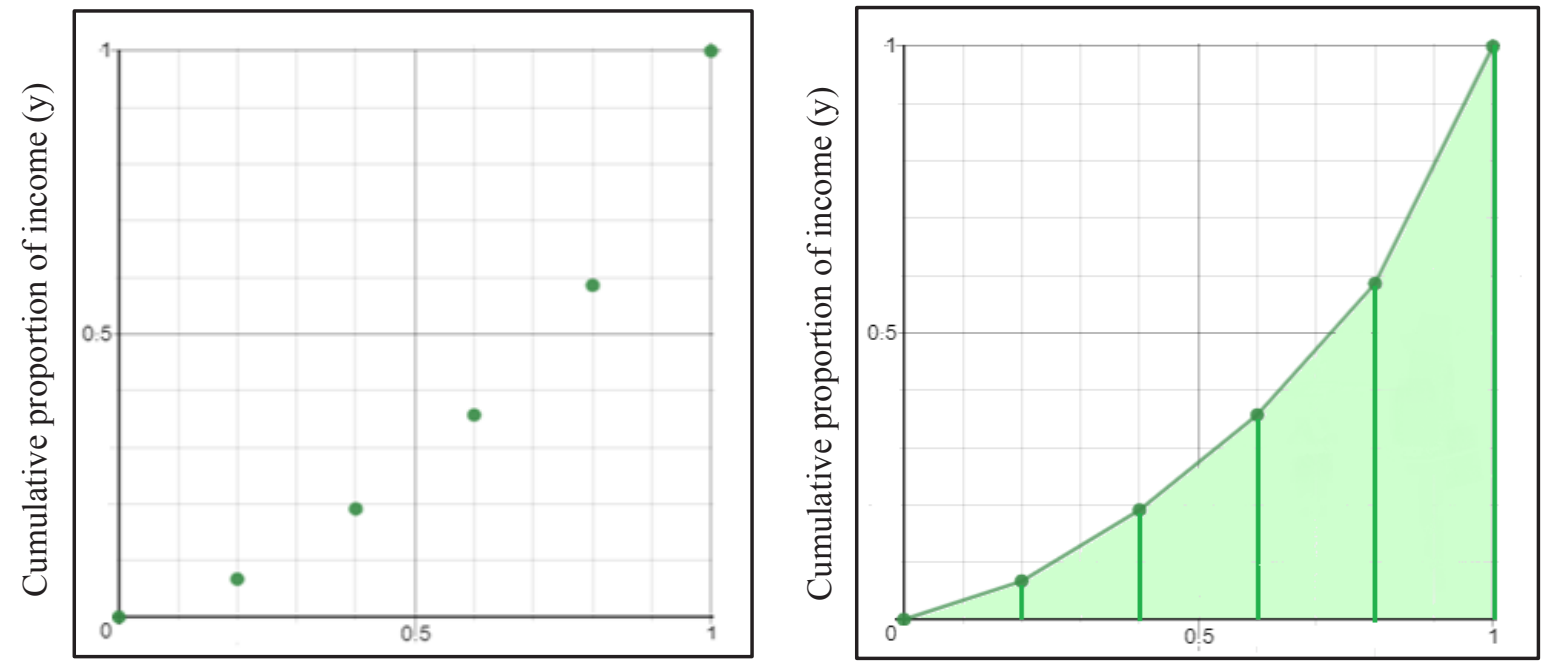

Figure 4. Using the Trapezoid Rule to approximate the area under the Lorenz curve representnig Canada's 2017 income distribution.

which is used to approximate the definite integral, is given by equation 4.6.

$$
A=\frac{h}{2}\left(b_{1}+b_{2}\right)
$$

where:

$$
\begin{aligned}
& b_{1}=\text { first base of the trapezoid } \\
& b_{2}=\text { second base of the trapezoid } \\
& h=\text { height of trapezoid }
\end{aligned}
$$

$$
\begin{gathered}
\int_{0}^{1} L(x) d x \approx \frac{h}{2}\left(y_{0}+y_{1}\right)+\frac{h}{2}\left(y_{1}+y_{2}\right)+\frac{h}{2}\left(y_{2}+y_{3}\right)+\frac{h}{2}\left(y_{3}+y_{4}\right)+\frac{h}{2}\left(y_{4}+y_{5}\right) \\
\int_{0}^{1} L(x) d x \approx \frac{h}{2}\left[y_{0}+2 y_{1}+2 y_{2}+2 y_{3}+2 y_{4}+y_{5}\right] \\
\int_{0}^{1} L(x) d x \approx \frac{0.2}{2}[0+2(0.067)+2(0.191)+2(0.357)+2(0.586)+1.000] \\
\int_{0}^{1} L(x) d x \approx 0.340
\end{gathered}
$$

At last, the Gini index is evaluated.

Using the data points from Table 2, the definite integral is approximated. 


$$
\begin{gathered}
G=1-2 \int_{0}^{1} L(x) d x \\
G \approx 1-2 \times 0.340 \\
\therefore G \approx \mathbf{G} . \mathbf{3 2 0}
\end{gathered}
$$

Integration. Using Logger Pro technology, the curve of best fit for the data is approximated using polynomial functions. The possible curves of best fit and their corresponding correlation coefficients are summarized in Table 3.

A correlation coefficient close to 1 provides a more accurate function to represent the data. Seeing that a fourthdegree polynomial provides the highest possible correlation coefficient, the quartic function is substituted into equation 4.6, and the Gini index is evaluated.

$$
\begin{gathered}
G=1-2 \int_{0}^{1} L(x) d x \\
G \approx 1-2 \int_{0}^{1}\left(1.784 x^{4}-2.754 x^{3}+1.933 x^{2}+0.03652 x+0.0002579\right) \\
G \approx 1-2\left[0.3568 x^{5}-0.6885 x^{4}+\frac{1.933}{3} x^{3}+0.01826 x^{2}+0.0002579 x\right]_{0}^{1} \\
G \approx 1-2\left[0.3568-0.6885+\frac{1.933}{3}+0.01826+0.0002579\right]_{0}^{1} \\
\therefore \boldsymbol{G} \approx \mathbf{0 . 3 3 8}
\end{gathered}
$$

\section{RESULTS}

Table 4 summarizes the Gini indices obtained for the 1999 , 2010, and 2017 Canadian income distributions using the three approaches outlined in the paper.
Table 4. 1999, 2010, and 2017 Canadian Gini indices evaluated using three of the earliest developed methods

\begin{tabular}{cccc} 
& $\mathbf{1 9 9 9}$ & $\mathbf{2 0 1 0}$ & $\mathbf{2 0 1 7}$ \\
Original & 0.410 & 0.410 & 0.411 \\
$\begin{array}{c}\text { Relative Mean Difference } \\
\text { Geometric (Trapezoid } \\
\text { Rule) }\end{array}$ & 0.410 & 0.410 & 0.411 \\
$\begin{array}{c}\text { Geometric (Logger Pro) } \\
\text { (Lom }\end{array}$ & 0.330 & 0.318 & 0.320 \\
\hline
\end{tabular}

As highlighted above, the original and the relative mean difference approaches, collectively referred to as the algebraic methods, provided identical results when applied to three different data sets. Since the two methods provided the same results, they can be used interchangeably with the data sets analyzed in this paper. In contrast, the Gini values obtained using the geometric methods were different from the algebraic values and differed between the subtypes of the geometric method. It is also worth noting that the Trapezoid Rule will always produce a smaller Gini value than its geometric counterpart. This is because the Trapezoid Rule over-approximates the area under a Lorenz curve due to its use of trapezoids while the Logger Pro method uses curves that are better fits for the approximation. As a result, after doubling the area and subtracting it from one (i.e. implementing the equation for the Gini Index), a smaller Gini value is produced by the Trapezoid Rule. As for generalizing a trend for the results of the arithmetic and geometric methods, the calculations from this paper suggest that the geometric methods will always produce smaller Gini values than the arithmetic methods. However, data from additional samples are needed in order to verify this generalization.

Table 3. Curves of best fit approximated by Logger Pro for the data in Table 2

Degree

Equation for $L(x)$
Correlation Coefficient

0.9574

0.9976

0.9995

1.000 
Moreover, to emphasize the differences between the four methods, the percentage differences between the algebraic and the geometric values of each data set are provided in Table 5. The calculations involved in evaluating the percentage difference have been shown for the 2017 income distribution. Note that the evaluation of percentage difference is an appropriate procedure for this comparison since the Gini values within each data set are independent measurements that represent the same data.

$$
\% \text { difference }=\frac{\text { Difference }}{\text { Average }} \times 100 \%
$$

Geometric (Trapezoid Rule) \& Algebraic:

$$
\% \text { difference }=\frac{0.411-0.320}{\frac{0.411+0.320}{2}} \times 100 \% \approx 24.9 \%
$$

Geometric (Technology) \& Algebraic:

$$
\% \text { difference }=\frac{0.411-0.338}{\frac{0.411+0.338}{2}} \times 100 \% \approx 19.5 \%
$$

The percentage differences for each data set as well as the average percentage differences demonstrate the contrast between the Gini values obtained by the algebraic and geometric methods. This difference is due to the reliance of the geometric methods on a Lorenz curve. and the use of either geometric shapes or technology to approximate the area under the curve. Since these approximations are then used to evaluate the Gini index, contrasting Gini indices are obtained when using the geometric methods.

Table 5. Percentage differences between the Gini values of the geometric and algebraic approaches for the Canadian income distributions in 1999, 2010, and 2017.

$\begin{array}{ccccc} & \mathbf{1 9 9 9} & \mathbf{2 0 1 0} & \mathbf{2 0 1 7} & \text { Average } \\ \begin{array}{c}\text { Geometric } \\ \text { (Trapezoid Rule) } \\ \text { \& Algebraic }\end{array} & 27.1 \% & 25.3 \% & 24.9 \% & 25.8 \% \\ \begin{array}{c}\text { Geometric } \\ \text { (Logger Pro) \& } \\ \text { Algebraic }\end{array} & 21.6 \% & 20.1 \% & 19.5 \% & 20.4 \% \\ & & & & \\ \end{array}$

\section{CONCLUSION}

Using the Canadian income distribution from 1999, 2010, and 2017, it was discovered that Gini's original method and the relative mean difference method, referred to as the algebraic methods, provide identical Gini indices for the analyzed data sets. In contrast, the geometric methods, referring to the Trapezoid Rule and Logger Pro technology, provide Gini indices that differed from one another and the algebraic methods. Because of the global use of the Gini index and how different values can be obtained depending on the chosen approach, it becomes important to acknowledge the method being used. If the geometric approaches are chosen, it is essential to address the exact methodology since each method can provide a different value. Currently, most websites and organizations, such as Wikipedia, World Bank, and The World Factbook, provide no information on the methods used to evaluate the Gini Index. As is often the case with mathematical models, most of these sources place a majority of the emphasis on the final result rather than the method applied. However, as highlighted in this paper, the methods provide crucial insight and must be acknowledged along with their final result in order to allow valid interpretation.

Moreover, the results of this investigation suggest that the algebraic methods are especially preferable when dealing with discrete data, which is often the case with reallife situations. However, if dealing with continuous data, the geometric methods are preferable since the algebraic approaches solely allow discrete data while the geometric approaches allow both discrete and continuous. The geometric approaches, then, are the only available option when dealing with continuous data.

Lastly, it is important to acknowledge that the discussion in this paper was limited to the three earliest methods developed for the evaluation of the Gini index. Numerous other approaches were later published for the popular Gini index, which are not analyzed in this paper. A few notable examples include the covariance method developed by Sudhir Anand (1983) [2], the matrix approach (1989) developed by Jacques Silber, and the mean approach developed by Anthony Shorrocks (1999) [4]. An interesting extension is to investigate these other methods and analyze how the produced Gini Indices compare to one another for the same data set. This will then determine which methods are interchangeable 
with one another. Knowing this will allow countries to use the methods that are consistent with each other for their respective income distributions and will consequently allow a more accurate comparison between the Gini values of different regions as well as a more accurate global ranking.

\section{REFERENCES}

1. Ceriani, L., \& Verme, P. (2012). The origins of the Gini index: extracts from Variabilità e Mutabilità (1912) by Corrado Gini. The Journal of Economic Inequality, 10(3), 422-443. https://doi.org/10.1007/s10888-011-9188-x

2. Xu, K. (2003). How has the Literature on Gini's Index Evolved in the Past 80 Years?. Retrieved from https:// papers.ssrn.com/sol3/papers.cfm?abstract_id=423200

3. The World Bank. GINI index (World Bank estimate) [Internet]. Data.worldbank.org. 2019. Retrieved from: https://data.worldbank.org/indicator/si.pov.gini?most_ recent_value_desc=false $\&$ view=map

4. Ceriani, L., \& Verme, P. (2014). Working Paper Series: Individual Diversity and the Gini Decomposition (Publication No. 321). Retrieved from ECINEQ website: http://www.ecineq.org/milano/WP/ECINEQ2014-321.pdf

5. Gini, C. (1912). Indici di variabilità. In C. Gini (Author), Variabilita e mutabilita (pp. 23-119). Retrieved from Byterfly database.

6. Dalton, H. (1920). The measurement of the inequality of incomes. The Economic Journal, 30(119), 348-361. https:// doi.org/10.2307/2223525

7. Kendall, M., \& Stuart, A. (1958). The Advanced Theory of Statistics: Distribution Theory (Vol. 1). New York Hafner Publishing Company.

8. Lows, R. (1984). Measurement of inequality: The Gini coefficient and school finance studies. Journal of Education Finance, 10(1), 83-94. Abstract retrieved from JSTOR database.

9. Lorenz, M. (1905). Methods of Measuring the Concentration of Wealth. American Statistical Association, 9(70), 209-219. https://doi. org/10.2307/2276207

10. Statistics Canada. (2020, April 22). Distribution of disposable income for each household group, selected years [Table]. Retrieved from https://www150.statcan. gc.ca/n1/daily-quotidien/180322/t002b-eng.htm

11. Stewart, J. (2012). Calculus: Early Transcendentals (7th ed., pp. 506-511). Cengage Learning. 


\section{APPENDIX A}

\section{Data, calculations, and Gini indices for the 1999 Canadian income distribution}

Table 6. 1999 Canadian income distribution [10]

Income Quintile

Cumulative Proportion of Population

$$
\boldsymbol{x}_{\boldsymbol{p}}
$$

$1^{\text {st }}$ Quintile

$2^{\text {nd }}$ Quintile

$3^{\text {rd }}$ Quintile

$4^{\text {th }}$ Quintile

$5^{\text {th }}$ Quintile

Original Method:

$$
\begin{gathered}
\mu_{y}=\frac{0.066+0.192+0.365+0.596+1.000}{5}=0.4438 \\
G=\frac{1}{\mu_{y} n^{2}} \sum_{i=1}^{\frac{n+1}{2}}(n+1-2 i)\left(y_{n-i+1}-y_{i}\right) \\
G=\frac{1}{11.095} \sum_{i=1}^{3}(6-2 i)\left(y_{6-i}-y_{i}\right) \\
G=\frac{1}{11.095}[4(1.000-0.066)+2(0.596-0.192)+0] \\
\therefore \boldsymbol{G} \approx \mathbf{0 . 4 1 0}
\end{gathered}
$$

Relative Mean Difference Method:

$$
A M D=\sum_{i=1}^{n} \sum_{j=1}^{n}\left|x_{i}-y_{j}\right|
$$

$A M D=|0.066-0.066|+|0.066-0.192|+|0.0660 .365|+|0.066-0.596|+|0.066-1.000|+|0.192-0.066|+\mid 0.192-$ $0.192|+| 0.192-0.365|+| 0.192-0.596|+| 0.192-1.000|+| 0.365-0.066|+| 0.365-0.192|+| 0.365-0.365|+| 0.365-$ $0.596|+| 0.365-1.000|+| 0.596-0.066|+| 0.596-0.192|+| 0.586-0.365|+| 0.596-0.596|+| 0.596-1.000|+| 1.000-$ $0.066|+| 1.000-0.192|+| 1.000-0.365|+| 1.000-0.596|+| 1.000-1.000 \mid$

$$
A M D=9.088
$$




$$
\begin{gathered}
G=\frac{\sum_{i=1}^{n} \sum_{j=1}^{n}\left|x_{i}-y_{j}\right|}{2 n^{2} \mu_{y}} \\
G=\frac{9.088}{2 \times 25 \times 0.4438} \\
\therefore \boldsymbol{G} \approx \mathbf{0 . 4 1 0}
\end{gathered}
$$

Geometric Method - Trapezoid Rule:

$$
\begin{gathered}
\int_{0}^{1} L(x) d x \approx \frac{h}{2}\left[y_{0}+2 y_{1}+2 y_{2}+2 y_{3}+2 y_{4}+y_{5}\right] \\
\int_{0}^{1} L(x) d x \approx \frac{0.2}{2}[0+2(0.066)+2(0.192)+2(0.365)+2(0.596)+1.000] \\
\int_{0}^{1} L(x) d x \approx 0.344 \\
G=1-2 \int_{0}^{1} L(x) d x \\
G \approx 1-2 \times 0.344 \\
\therefore \boldsymbol{G} \approx \mathbf{0 . 3 1 2}
\end{gathered}
$$

Geometric Method - Logger Pro Technology:

$$
\begin{gathered}
L(x)=1.667 x^{4}-2.641 x^{3}+1.951 x^{2}+0.02251 x+0.0003175 \\
G=1-2 \int_{0}^{1} L(x) d x \\
G \approx 1-2 \int_{0}^{1}\left(1.667 x^{4}-2.641 x^{3}+1.951 x^{2}+0.02251 x+0.0003175\right) \\
G \approx 1-2\left[0.3334 x^{5}-0.66025 x^{4}+\frac{1.951}{3} x^{3}+0.011255 x^{2}+0.0003175 x\right]_{0}^{1} \\
\therefore G \approx \mathbf{0 . 3 3 0}
\end{gathered}
$$




\section{APPENDIX B}

\section{Data, calculations, and Gini indices for the 2010 Canadian income distribution}

Table 7. 2010 Canadian income distribution [10]

\section{Cumulative Proportion of} Population

Income Quintile

$1^{\text {st }}$ Quintile

$2^{\text {nd }}$ Quintile

$3^{\text {rd }}$ Quintile

$4^{\text {th }}$ Quintile

$5^{\text {th }}$ Quintile

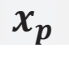

0.2

0.4

0.6

0.8

1.0
Cumulative Proportion of Income

Proportion of Total Income

0.067

0.124

0.169

0.360

0.228

0.412
0.067

0.191

0.588

\section{$y_{p}$}

1.000

Original Method:

$$
\begin{gathered}
\mu_{y}=\frac{0.067+0.191+0.360+0.588+1.000}{5}=0.4412 \\
G=\frac{1}{\mu_{y} n^{2}} \sum_{i=1}^{\frac{n+1}{2}}(n+1-2 i)\left(y_{n-i+1}-y_{i}\right) \\
G=\frac{1}{11.03} \sum_{i=1}^{3}(6-2 i)\left(y_{6-i}-y_{i}\right) \\
G=\frac{1}{11.03}[4(1.000-0.067)+2(0.588-0.191)+0] \\
\therefore \boldsymbol{G} \approx \mathbf{0 . 4 1 0}
\end{gathered}
$$

Relative Mean Difference Method:

$$
A M D=\sum_{i=1}^{n} \sum_{j=1}^{n}\left|x_{i}-y_{j}\right|
$$

$A M D=|0.067-0.067|+|0.067-0.191|+|0.067-0.360|+|0.067-0.588|+|0.067-1.000|+|0.191-0.067|+|0.191-0.191|+\mid 0.191-$ $0.305|+| 0.191-0.588|+| 0.191-1.000|+| 0.360-0.067|+| 0.360-0.191|+| 0.360-0.360|+| 0.360-0.588|+| 0.360-1.000|+| 0.588-$ $0.067|+| 0.588-0.191|+| 0.588-0.360|+| 0.588-0.588|+| 0.588-1.000|+| 1.000-0.067|+| 1.000-0.191|+| 1.000-0.360|+| 1.000-$

$0.588|+| 1.000-1.000 \mid$

$$
A M D=9.052
$$




$$
\begin{gathered}
G=\frac{\sum_{i=1}^{n} \sum_{j=1}^{n}\left|x_{i}-y_{j}\right|}{2 n^{2} \mu_{y}} \\
G=\frac{9.052}{2 \times 25 \times 0.4412} \\
\therefore \boldsymbol{G} \approx \mathbf{0 . 4 1 0}
\end{gathered}
$$

Geometric Method - Trapezoid Rule:

$$
\begin{gathered}
\int_{0}^{1} L(x) d x \approx \frac{h}{2}\left[y_{0}+2 y_{1}+2 y_{2}+2 y_{3}+2 y_{4}+y_{5}\right] \\
\int_{0}^{1} L(x) d x \approx \frac{0.2}{2}[0+2(0.067)+2(0.191)+2(0.360)+2(0.588)+1.000] \\
\int_{0}^{1} L(x) d x \approx 0.341 \\
G=1-2 \int_{0}^{1} L(x) d x \\
G \approx 1-2 \times 0.341 \\
\therefore \boldsymbol{G} \approx \mathbf{0 . 3 1 8}
\end{gathered}
$$

Geometric Method - Logger Pro Technology:

$$
\begin{gathered}
L(x)=1.784 x^{4}-2.784 x^{3}+1.972 x^{2}+0.02757 x+0.0003373 \\
G=1-2 \int_{0}^{1} L(x) d x \\
G \approx 1-2 \int_{0}^{1}\left(1.784 x^{4}-2.784 x^{3}+1.972 x^{2}+0.02757 x+0.0003373\right) \\
G \approx 1-2\left[0.3568 x^{5}-0.696 x^{4}+\frac{1.972}{3} x^{3}+0.013785 x^{2}+0.0003373 x\right]_{0}^{1} \\
\therefore G \approx \mathbf{0 . 3 3 5}
\end{gathered}
$$




\section{APPENDIX C}

\section{Evaluating the cumulative proportion of population and the cumulative proportion of income for an arbitrary population}

Consider a population of four individuals, with each individual earning the income specified in Table 8. Summing the incomes of the four individuals provides $\$ 20.00$ as the total income of the population.

Table 8. An arbitrary population composed of 4 individuals and their corresponding incomes

Individual

Income
1

$\$ 2.00$
2

$\$ 3.00$
3

$\$ 5.00$
4

$\$ 10.00$

Using equation 1.1, the cumulative proportion of population up to each individual is evaluated as shown.

$$
x_{1}=\frac{1}{4}=0.25 \quad x_{2}=\frac{2}{4}=0.50 \quad x_{3}=\frac{3}{4}=0.75 . \quad x_{4}=\frac{4}{4}=1.00
$$

Similarly, using equation 2.2 , the cumulative proportion of income up to each individual is evaluated as shown.

$$
\begin{array}{cc}
y_{1}=\frac{\$ 2.00}{\$ 20.00}=0.10 & y_{2}=\frac{\$ 2.00+\$ 3.00}{\$ 20.00}=0.25 \\
y_{3}=\frac{\$ 2.00+\$ 3.00+\$ 5.00}{\$ 20.00}=0.50 & y_{4}=\frac{\$ 2.00+\$ 3.00+\$ 5.00+\$ 10.00}{\$ 20.00}=1.00
\end{array}
$$

Pairing up each $x$-coordinate with its corresponding $y$-coordinate will then provide the following four points for evaluating the Gini Index. Note that the origin $(0,0)$ is also considered when evaluating the Gini Index.

$$
(0.25,0.10) \quad(0.50,0.25) \quad(0.75,0.50) \quad(1.00,1.00)
$$

\section{Appendix D}

\section{Equality between Dalton's statement and Gini's mean difference $(\Delta)$}

To understand the equality between Dalton's definition of $\Delta$ and Gini's equation for $\Delta$, consider sequence 5.1, which has its $n$ terms arranged in an ascending order.

$$
y_{1}, y_{2}, \ldots, y_{n-2}, y_{n-1}, y_{n}
$$

The sum of the non-negative differences between all possible pairs is taken. 


$$
\begin{gathered}
\left(y_{1}-y_{1}\right)+\left(y_{2}-y_{1}\right)+\left(y_{3}-y_{1}\right)+\cdots+\left(y_{n-1}-y_{1}\right)+\left(y_{n}-y_{1}\right) \\
+\left(y_{2}-y_{2}\right)+\left(y_{3}-y_{1}\right)+\cdots+\left(y_{n-1}-y_{2}\right)+\left(y_{n}-y_{2}\right) \\
+\left(y_{3}-y_{3}\right)+\cdots+\left(y_{n-1}-y_{3}\right)+\left(y_{n}-y_{3}\right) \\
\cdots \\
+\left(y_{n-1}-y_{n-1}\right)+\left(y_{n}-y_{n-1}\right) \\
+\left(y_{n}-y_{n}\right)
\end{gathered}
$$

After cancelling all zero pairs, the terms are rearranged by considering the number of times each term occurs in the sum. For example, the term $y_{n}$ occurs $(n-1)$ times, $y_{n-1}$ occurs $(n-2)$ times, and so on.

$$
\begin{gathered}
(n-1)\left(-y_{1}\right)+(n-2)\left(-y_{2}\right)+\cdots+2\left(-y_{n-2}\right)+1\left(-y_{n}\right) \\
+1\left(y_{2}\right)+2\left(y_{3}\right)+\cdots+(n-2)\left(y_{n-1}\right)+(n-1)\left(y_{n}\right)
\end{gathered}
$$

From the above, like terms are factored out and the sum is rewritten. For example, from factoring out $-y_{2}$ from $(n-2)\left(-y_{2}\right)$ and $\left(y_{2}\right)$, the term $-y_{2}(n-3)$ is obtained, and from factoring out $y_{n-1}$ from $(n-2)\left(y_{n-1}\right)$ and $\left(y_{n-1}\right)$, the term $y_{n-1}(n-3)$ is obtained. Note that the coefficient of $n$ in each term is intentionally kept positive.

$$
\begin{gathered}
-y_{1}(n-1)-y_{2}(n-3)-y_{3}(n-5)-\cdots-\frac{y_{\frac{n+1}{2}}}{2}(n+1-2 i) \\
+y_{\frac{n+1}{2}}(n+1-2 i)+\cdots+y_{n-2}(n-5)+y_{n-1}(n-3)+y_{n}(n-1)
\end{gathered}
$$

Once again, like terms are factored out.

$$
(n-1)\left(y_{n}-y_{1}\right)+(n-3)\left(y_{n-1}-y_{2}\right)+\cdots+(n+1-2 i)\left(y_{\frac{n+1}{2}}-y_{\frac{n+1}{2}}\right)
$$

The sum is then expressed using sigma notation.

$$
\sum_{i=1}^{\frac{n+1}{2}}(n+1-2 i)\left(y_{n-i+1}-y_{i}\right)
$$

Since the absolute value of the negative differences equals the positive differences, the above expression is multiplied by 2 to account for all the possible differences when taken positively. Lastly, since there are $n$ possible values for the first and second term in the difference, the expression is divided by $n^{2}$ to consider the average of the sum. Based on Dalton's definition, this provides an equation for Gini's mean difference, which matches the equation proposed by Gini in his book [5]. 


$$
\Delta=\frac{2}{n^{2}} \sum_{i=1}^{\frac{n+1}{2}}(n+1-2 i)\left(y_{n-i+1}-y_{i}\right)
$$

From translated extracts of the Italian text, the Gini index is defined as the Gini's mean difference "divided by twice the arithmetic mean" [4].

$$
G=\frac{\Delta}{2 \mu_{y}}
$$

After substituting equation 5.3 into equation 5.4, the equation for the Gini index based on the original definition is obtained.

$$
G=\frac{\Delta}{2 \mu_{y}}=\frac{1}{\mu_{y} n^{2}} \sum_{i=1}^{\frac{n+1}{2}}(n+1-2 i)\left(y_{n-i+1}-y_{i}\right)
$$

\section{Appendix E}

\section{Equality between the original and adjusted versions of the Gini index}

To understand the equality between the original and adjusted equations, consider the ascending data from sequence 5.1 and the sum of the negative differences between all the possible pairs.

$$
\begin{gathered}
\left(y_{1}-y_{2}\right) \\
+\left(y_{1}-y_{3}\right)+\left(y_{2}-y_{3}\right) \\
+\left(y_{1}-y_{4}\right)+\left(y_{2}-y_{4}\right)+\left(y_{3}-y_{4}\right) \\
\cdots \\
\left(y_{1}-y_{n-1}\right)+\left(y_{2}-y_{n-1}\right)+\left(y_{3}-y_{n-1}\right)+\cdots+\left(y_{n-2}-y_{n-1}\right) \\
\left(y_{1}-y_{n}\right)+\left(y_{2}-y_{n}\right)+\left(y_{3}-y_{n}\right)+\cdots+\left(y_{n-1}-y_{n}\right)
\end{gathered}
$$

The terms are again rearranged by cnsidering the number of times each term occurs in the sum. Like terms are then factored out twice as done before and the sum is rewritten.

$$
(n-1)\left(y_{1}-y_{n}\right)+(n-3)\left(y_{2}-y_{n-1}\right)+(n-5)\left(y_{3}-y_{n-2}\right)+\cdots+(n+1-2 i)\left(\frac{y_{\frac{n+1}{2}}}{2}-y_{\frac{n+1}{2}}\right)
$$

Since Gini's mean difference is a real-life application that consequently requires positive differences, each term in the above sum is multiplied by negative one.

$$
(1-n)\left(y_{1}-y_{n}\right)+(3-n)\left(y_{2}-y_{n-1}\right)+(5-n)\left(y_{3}-y_{n-2}\right)+\cdots+(n+1-2 i)\left(\frac{y_{\frac{n+1}{2}}}{-}-y_{\frac{n+1}{2}}\right)
$$

Thus, adding the above differences to the positive differences in expression 5.2 provides an alternative expression for the sum of all possible differences when taken positively. 


$$
\begin{gathered}
(n-1)\left(y_{n}-y_{1}\right)+(n-3)\left(y_{n-1}-y_{2}\right)+\cdots+(n+1-2 i)\left(y_{\frac{n+1}{2}}-y_{\frac{n+1}{2}}\right)+\cdots \\
+(3-n)\left(y_{2}-y_{n-1}\right)+(1-n)\left(y_{1}-y_{n}\right)
\end{gathered}
$$

The above sum is then written using sigma notation and divided by $n^{2}$ to obtain an alternative equation for Gini's mean difference that assumes $n$ to be any whole number.

$$
\Delta=\frac{\sum_{i=1}^{n}(n+1-2 i)\left(y_{n-i+1}-y_{i}\right)}{n^{2}}
$$

Finally, substituting equation 5.5 for $\Delta$ in equation 5.4 provides an adjusted equation for the Gini index that is interchangeable with the original equation.

$$
G=\frac{\Delta}{2 \mu_{y}}=\frac{1}{2 \mu_{y} n^{2}} \sum_{i=1}^{n}(n+1-2 i)\left(y_{n-i+1}-y_{i}\right)
$$

\section{Appendix F}

\section{Use of original Gini Equation for the 2017 Canadian income distribution}

Using the original Gini equation, the same Gini value as the one from the adjusted equation is obtained since the two equations are interchangeable. This can be proven by applying the original Gini equation, as provided below, to the 2017 Canadian income distribution.

$$
\begin{gathered}
G=\frac{1}{\mu_{y} n^{2}} \sum_{i=1}^{\frac{n+1}{2}}(n+1-2 i)\left(y_{n-i+1}-y_{i}\right) \\
G=\frac{1}{\mu_{y} n^{2}} \sum_{i=1}^{\frac{n+1}{2}}(n+1-2 i)\left(y_{n-i+1}-y_{i}\right) \\
G=\frac{1}{11.005} \sum_{i=1}^{3}(6-2 i)\left(y_{6-i}-y_{i}\right) \\
\begin{array}{c}
11.005 \\
{[4}
\end{array} \\
G=\frac{1}{11.005}[3.732+0.790+0] \\
G=\frac{4.522}{11.005}
\end{gathered}
$$


As shown above, the Gini index from the original equation matches the value obtained using the adjusted equation, highlighting their interchangeable nature.

\section{Appendix G}

Calculating the absolute mean difference (AMD) using the 2017 Canadian income distribution

$$
\begin{gathered}
A M D=\sum_{i=1}^{n} \sum_{j=1}^{n}\left|x_{i}-y_{j}\right| \\
A M D=|0.067-0.067|+|0.067-0.191|+|0.067-0.357|+|0.067-0.586|+ \\
|0.067-1.000|+|0.191-0.067|+|0.191-0.191|+|0.191-0.357|+\mid 0.191- \\
0.586|+| 0.191-1.000|+| 0.357-0.067|+| 0.357-0.191|+| 0.357-0.357 \mid+ \\
|0.357-0.586|+|0.357-1.000|+|0.586-0.067|+|0.586-0.191|+|0.586-0.357|+ \\
|0.586-0.586|+|0.586-1.000|+|1.000-0.067|+|1.000-0.191|+|1.000-0.357|+ \\
\quad|1.000-0.586|+|1.000-1.000| \\
\begin{array}{c}
0.166+0+0.229+0.643+0.519+0.395+0.229+0+0.414+0.933 \\
+0.809+0.643+0.414+0
\end{array} \\
\therefore A M D=9.044
\end{gathered}
$$

